\title{
Prevalence of and risk factors for fatty liver in the general population of Northern Italy: the Bagnacavallo Study
}

Francesco Giuseppe Foschi ${ }^{1 \dagger}$, Giorgio Bedogni ${ }^{2 \dagger}$, Marco Domenicali ${ }^{3}$, Pierluigi Giacomoni ${ }^{4}$, Anna Chiara Dall'Aglio ${ }^{1}$, Francesca Dazzani ${ }^{1}$, Arianna Lanzi ${ }^{1}$, Fabio Conti ${ }^{3,5^{*}}$, Sara Savini ${ }^{1}$, Gaia Saini ${ }^{1}$, Mauro Bernardi ${ }^{3}$, Pietro Andreone ${ }^{5}$, Amalia Gastaldelli ${ }^{6}$, Andrea Casadei Gardini ${ }^{7}$, Claudio Tiribelli ${ }^{2}$, Stefano Bellentani ${ }^{8}$

and Giuseppe Francesco Stefanini ${ }^{1}$

\begin{abstract}
Background: The estimation of the burden of disease attributable to fatty liver requires studies performed in the general population.

Methods: The Bagnacavallo Study was performed between October 2005 and March 2009. All the citizens of Bagnacavallo (Ravenna, Italy) aged 30 to 60 years as of January 2005 were eligible. Altered liver enzymes were defined as alanine transaminase $>40 \mathrm{U} / \mathrm{l}$ and/or aspartate transaminase $>37 \mathrm{U} / \mathrm{l}$.

Results: Four thousand and thirty-three (58\%) out of 6920 eligible citizens agreed to participate and 3933 (98\%) had complete data. 393 (10\%) of the latter had altered liver enzymes and 3540 had not. After exclusion of subjects with HBV or HCV infection, liver ultrasonography was available for $93 \%$ of subjects with altered liber enzymes and $52 \%$ of those with normal liver enzymes. The prevalence of fatty liver, non-alcoholic fatty liver disease (NAFLD) and alcoholic fatty liver disease (AFLD) was $0.74(95 \% \mathrm{Cl} 0.70$ to 0.79$)$ vs. 0.35 (0.33 to 0.37$), 0.46$ (0.41 to 0.51 ) vs. 0.22 (0.21 to 0.24$)$ and 0.28 (0.24 to 0.33 ) vs. 0.13 (0.11 to 0.14 ) in citizens with than in those without altered liver enzymes. Ethanol intake was not associated and all the components of the metabolic syndrome (MS) were associated with fatty liver. All potential risk factors were associated with a lower odds of normal liver vs. NAFLD while they were unable to discriminate AFLD from NAFLD.
\end{abstract}

Conclusions: Fatty liver as a whole was highly prevalent in Bagnacavallo in 2005/9 and was more common among citizens with altered liver enzymes.

Keywords: epidemiology, cross-sectional study, prevalence, risk factors, fatty liver, chronic liver disease

\section{Background}

Fatty liver (FL), the most common liver disease worldwide, is usually classified into non-alcoholic fatty liver disease (NAFLD) and alcoholic fatty liver disease (AFLD) $[1,2]$. After the exclusion of hepatitis B and C and steatogenic drugs, NAFLD is currently diagnosed

\footnotetext{
* Correspondence: fabio.conti2@studio.unibo.it

${ }^{\dagger}$ Francesco Giuseppe Foschi and Giorgio Bedogni contributed equally to this work.

${ }^{3}$ Department of Medical and Surgical Sciences, University of Bologna, Via Massarenti 9, 40138 Bologna, Italy

${ }^{5}$ Research Center for the Study of Hepatitis, Department of Medical and Surgical Sciences, University of Bologna, Bologna, Italy

Full list of author information is available at the end of the article
}

when $\mathrm{FL}$ is associated with an ethanol intake $\leq 20 \mathrm{~g} /$ day in women and $\leq 30 \mathrm{~g} /$ day in men $[1,2]$. The NAFLD vs. AFLD dichotomization is useful in clinical practice because ethanol is unlikely to be toxic at quantities $\leq 30 \mathrm{~g} /$ day but hides the important fact that ethanol and obesity do interact to determine the burden of liver disease in the general population [2-4]. FL is commonly considered the hepatic manifestation of the metabolic syndrome (MS) but the formal testing of the hypothesis that FL is the hepatic component of MS has led to conflicting results [2, 5-7].

In the early 2000s, the Dionysos Study reported the first data on the prevalence and incidence of FL in the

(c) The Author(s). 2019 Open Access This article is distributed under the terms of the Creative Commons Attribution 4.0 International License (http://creativecommons.org/licenses/by/4.0/), which permits unrestricted use, distribution, and 
general population $[8,9]$. In the Dionysos Nutrition \& Liver Study, the citizens of Campogalliano (Modena, Emilia-Romagna, Italy) with suspected liver disease were matched with randomly chosen citizens without suspected liver disease to obtain estimates of the prevalence of and the risk factors for NAFLD and AFLD in the general population [8]. Many epidemiological studies on FL have been published since the Dionysos Nutrition \& Liver Study findings were made available [10]. The worldwide prevalence of NAFLD was estimated to be 0.25 (95\%CI 0.22 to 0.28 ) by a recent meta-analysis of 45 studies [10]. Eleven of these 45 studies were performed in Europe and yielded an estimate of 0.24 (0.16 to 0.33 ) for the prevalence of NAFLD. Five of these 11 studies used imaging techniques to diagnose FL and were performed in the general population [3, 8, 11-13], with one of them being a nested case-control study [3].

The so-called "ecology of medical care" model provides a strong rationale to expect that the estimates of illness made in the general population will differ from those obtained in other settings and this has indeed been repeatedly shown in practice $[14,15]$. The inescapable conclusion is that the real burden attributable to a given disease cannot be estimated without epidemiological data obtained from the general population [15]. There is also mounting evidence that within a given level of the ecology of medical care [14], the individuals actually studied are often not representative of the persons making up that level, e.g. the patients enrolled in trials of NAFLD drugs are not representative of those treated in everyday practice [16].

It was with the aim of providing data on the epidemiology of FL in the general population that we performed the Bagnacavallo Study of liver disease. In detail, the aim of the Bagnacavallo Study was threefold: 1) to evaluate the prevalence of and the risk factors for FL in a cross-section of the general population of a Northern Italy town; 2) to develop a cohort of subjects from the general population where the association between FL and incident health outcomes could be studied; 3) to develop a cohort of subjects from the general population where nested case-control studies of potential risk factors for FL could be performed (taking the advantage of a purposely built serum bank) [17].

The present report deals with the first aim of the Bagnacavallo Study, i.e. the estimation of the prevalence of and the risk factors for FL in a general population. We also report the prevalence of and the risk factors for FL dichotomized into NAFLD and AFLD.

\section{Methods}

\section{Study design}

The Bagnacavallo Study was performed between October 2005 and March 2009. All citizens of Bagnacavallo
(Ravenna, Emilia-Romagna, Italy) aged 30 to 60 years as of January 2005 were eligible and were invited by written letter to participate to the study. Public encounters were also held to promote participation to the study. Altered liver enzymes (ALE) were defined as alanine transaminase $($ ALT $)>40 \mathrm{U} / \mathrm{l}$ and/or aspartate transaminase $(\mathrm{AST})>37$ U/l. These cut-points were the upper normal limits of ALT and AST applied by the laboratory that performed all the analyses of the Bagnacavallo study. The study protocol specified that all ALE+ and at least $50 \%$ of ALE- citizens had to undergo liver ultrasonography (LUS). ALE- citizens were chosen consecutively on the basis of their surname starting from a randomly chosen letter of the alphabet. The study was approved by the Ethical Committee of Area Vasta Romagna - IRST (reference number 112). All citizens gave written informed consent.

\section{Clinical and anthropometric assessment}

All participants underwent a detailed clinical history and physical examination following the model of the Dionysos Study [8, 18]. Current alcohol intake was assessed by trained interviewers by measuring the quantity (grams) of beer, wine and liquor drunk in the week prior to the enrollment [19]. Such quantity was divided by 7 to obtain a daily estimate and converted into alcohol units with rounding to the next integer. The conversion was done using an alcohol unit corresponding to $10 \mathrm{~g}$ of ethanol. Weight and height were measured following international guidelines [20]. Body mass index (BMI) was calculated and classified following the NIH guidelines [21]. Waist circumference (WC) was measured at the midpoint between the last rib and the iliac crest [22].

\section{Laboratory assessment}

Venous blood samples were obtained after 12-h fasting. The performed blood tests included: 1) glucose; 2) triglycerides; 3) total cholesterol; 4) high-density lipoprotein (HDL) cholesterol; 5) low-density lipoprotein (LDL) cholesterol; 6) ALT; 7) AST; 8) gamma-glutamyl-transferase (GGT); 9) bilirubin; 10) hepatitis B surface antigen (HBsAg); 11) antibodies against hepatitis $\mathrm{C}$ virus (anti-HCV).

\section{Metabolic syndrome}

The MS was diagnosed using the harmonized international definition [23]. In detail, large $\mathrm{WC}$ was defined as $\mathrm{WC} \geq$ $102 \mathrm{~cm}$ in men and $\geq 88 \mathrm{~cm}$ in women; high triglycerides as triglycerides $\geq 150 \mathrm{mg} / \mathrm{dl}$ or use of triglyceride-lowering drugs; low HDL as HDL < $40 \mathrm{mg} / \mathrm{dl}$ in men and < $50 \mathrm{mg} / \mathrm{dl}$ in women or use of HDL-increasing drugs; high blood pressure as systolic blood pressure $\geq 130 \mathrm{mmHg}$ or diastolic blood pressure $\geq 85 \mathrm{mmHg}$ or use or blood pressure-lowering drugs; high glucose as glucose $\geq 100 \mathrm{mg} /$ $\mathrm{dl}$ or use of glucose lowering drugs; and MS as $\geq 3$ of the above. 


\section{Liver ultrasonography}

LUS was performed by five experienced physicians (ACDA, GS, FD, AL and FC) using the same methodology of the Dionysos Nutrition \& Liver Study [9, 24]. Normal liver was defined as the absence of liver steatosis or other liver abnormalities. Light FL was defined as the presence of slight "bright liver" or hepatorenal echo contrast without intrahepatic vessels blurring and no deep attenuation; moderate FL as the presence of mild "bright liver" or hepatorenal echo contrast without intrahepatic vessel blurring and with deep attenuation; and severe FL as diffusely severe "bright liver" or hepatorenal echo contrast, with intrahepatic vessels blurring (no visible borders) and deep attenuation without visibility of the diaphragm. NAFLD was defined as FL associated with ethanol intake $\leq 2$ alcohol units $(20 \mathrm{~g}) /$ day in women and $\leq 3$ alcohol units ( $30 \mathrm{~g}) /$ day in men testing negative for HBsAg and anti-HCV and not treated with steatogenic drugs [2]. AFLD was defined as FL associated with ethanol intake $\geq 2$ alcohol units / day in women and $\geq 3$ alcohol units / day in men testing negative for HBsAg and anti-HCV and not treated with steatogenic drugs [2].

\section{Statistical analysis}

Most continuous variables were not Gaussian-distributed and all are reported as median and interquartile range. Discrete variables are reported as the number and proportion of subjects with the characteristic of interest. Between-group comparisons of discrete variables were performed using Pearson's Chi-square test and those of continuous variables using median regression with heteroskedasticity-robust standard errors [25].

Binary logistic regression was used to evaluate the association between FL and potential risk factors by means of six pre-specified models $[26,27]$. The outcome of all the logistic regression models was FL (discrete; $0=$ no; 1 =yes). Model 1 had ALE $(0=$ no; $1=$ yes $)$ as predictor; Model 2 added sex $(0=$ female, $1=$ male $)$ and age (continuous, years/10) to Model 1; Model 3 added BMI (continuous, $\mathrm{kg} / \mathrm{m}^{2} / 5$ ) and ethanol intake (continuous, alcohol units) to Model 2; Model 4 replaced BMI in Model 3 with WC (continuous, cm/10); Model 5 added MS (discrete, $0=$ no; $1=$ yes) and ethanol intake (continuous, alcohol units) as predictors to Model 2; Model 6 replaced MS in Model 5 with its single components, i.e. large WC (discrete, $0=$ no; $1=$ yes), high triglycerides (discrete, $0=$ no; $1=$ yes), low HDL (discrete, $0=$ no; $1=$ yes), high blood pressure (discrete, $0=$ no; $1=$ yes) and high glucose (discrete, $0=$ no; $1=$ yes). Before fitting the logistic regression models, we used univariable and multivariable scatterplot smoothers to get an idea of the functional form and shape of the continuous predictors and found no evidence of deviation from linearity for any predictor [28]. We also checked that the multivariable logits of the continuous predictors were linear using multivariable fractional polynomials [29]. Because alcohol intake as quantified by the present study is strictly speaking an ordinal and not a continuous variable, we tested whether its multivariable relationship with $\mathrm{FL}$ was linear by modeling it as both continuous and discrete in the same model [30]. We found that the relationship was linear in all models (data not shown). We also tested whether age and gender interacted in the models involving them (Models 2 to 6) and found that they did not (data not shown). Even if ethanol was not associated with FL, we nonetheless evaluated its interaction with BMI and WC because of its potential clinical significance [3]. We found no evidence of interaction of ethanol with both BMI and WC (data not shown). We evaluated the presence of collinearity among predictors in all models using the Belsley-Kuh-Welsch condition number [31]. We compared models using Akaike information criterion (AIC) and the Bayesian information criterion (BIC) and additionally calculated Nagelkerke pseudo- $\mathrm{R}^{2}$ and the area the under the receiver-operating characteristic curve (-ROC-AUC) [26]. We used Model 3 to calculate and plot the sex-specific marginal probabilities of FL corresponding to the 5th, 25th, 50th, 75th and 95th internal percentiles of age and BMI at the median intake of ethanol [27, 32].

Multinomial logistic regression was used to evaluate the association between FL type and potential predictors by means of six pre-specified models [26, 27]. The outcome of all the multinomial logistic regression models was FL type (discrete; $0=$ NAFLD; $1=$ normal liver; $2=$ AFLD). The prediction models were the same described above under binary logistic regression with the exception that ethanol was not entered into any model. We set NAFLD as the reference category in order to obtain estimates of effect sizes for the normal liver vs. NAFLD and the AFLD vs. AFLD comparisons. We performed the same tests of model assumptions described above under binary logistic regression. We compared models using Akaike information criterion (AIC) and the Bayesian information criterion (BIC) and additionally calculated Nagelkerke pseudo-R ${ }^{2}$. ROC-AUC were calculated for the two binary logistic models underlying the multinomial logistic model. Statistical analysis was performed using Stata 15.1 (Stata Corporation, College Station, TX, USA).

\section{Results}

\section{Flow of the citizens through the study}

The flow of the citizens through the study is depicted in Fig. 1. Four thousand and thirty-three (58\%) of the 6920 citizens aged 30 to 60 years who resided in Bagnacavallo as of January 2005 agreed to participate to the study and 3933 (98\%) of them had all the data required for analysis. The citizens were consecutively studied during the first three days of every week (except for holidays) 


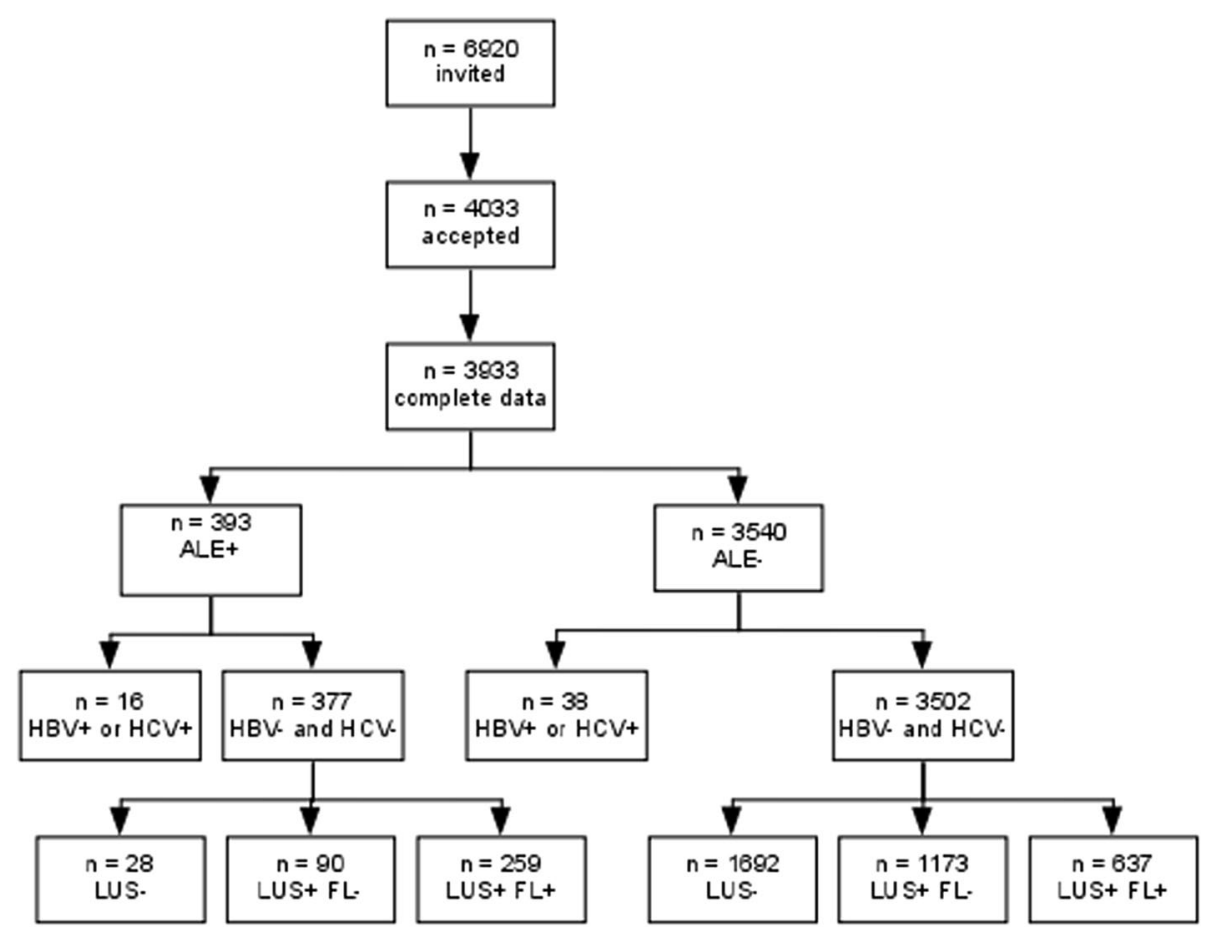

Fig. 1 Flow of the subjects through the study. Abbreviations: ALE = altered liver enzymes; HBV = Hepatitis B virus; HCV = hepatitis C virus; LUS = liver ultrasonography; $\mathrm{FL}=$ fatty liver

between October 2005 and March 2009. The study protocol required that all ALE+ and at least $50 \%$ of ALE- citizens were recalled to undergo LUS.

\section{Comparison of the subjects with and without liver ultrasonography among the citizens with altered liver enzymes}

Three hundred ninety-three (10\%) of the 3933 citizens with complete data were ALE+. Sixteen (4.1\%) of them had HBV or HCV infection and will not be considered here. Of the remaining 377 ALE+ citizens, 349 (93\%) underwent LUS. Additional file 1: Table S1 compares the 349 ALE+ citizens with LUS to the 28 ALE+ citizens without LUS. As compared to ALE+ citizens without LUS, ALE+ citizens with LUS had higher values of triglycerides, ALT and GGT $(p<0.05)$. Besides not being of great interest in itself [33], the lack of statistical significance should be taken with an additional degree of caution here owing to the low number of ALE+ subjects without LUS $(n=28)$.

\section{Comparison of the subjects with and without liver ultrasonography among the citizens with normal liver enzymes}

Among the 3540 (90\%) ALE- citizens, 38 had HBV or $\mathrm{HCV}$ infection (1.1\%) and will not be considered here. Of the remaining 3502 ALE- citizens, 1810 (52\%) underwent LUS. Additional file 2: Table S2 compares the 1810 ALE- citizens with LUS to the 1692 ALE- citizens without LUS. As compared to ALE- citizens without LUS, ALE- citizens with LUS were more likely to be male and had higher values of age, weight, BMI, WC, glucose, triglycerides, cholesterol, LDL-cholesterol, systolic blood pressure, ALT, GGT and bilirubin $(p \leq 0.05)$. Besides not being of great interest in itself [33], the presence of statistical significance should be taken with an additional degree of caution here owing to the high number of ALE- subjects with $(n=1810)$ and without LUS $(n=1692)$.

\section{Comparison of the citizens with and without altered liver} enzymes among those with liver ultrasonography

Table 1 compares the ALE+ and ALE- citizens with availability of LUS. ALE+ citizens were more frequently male and slightly younger than ALE- citizens. ALE+ citizens had greater values of BMI, WC, glucose, triglycerides, total cholesterol, LDL-cholesterol, systolic blood pressure and diastolic blood pressure and lower values of HDL-cholesterol.

\section{Prevalence and risk factors for fatty liver}

The prevalence of FL was 0.74 (95\%CI 0.70 to 0.79 ) among ALE+ and 0.35 (0.33 to 0.37) among ALE- citizens $(p<0.001)$. The severity of FL (light vs. moderate vs. severe) was also higher among ALE+ than ALE- citizens. All the components of the MS and the MS itself were more prevalent among ALE+ than ALE- subjects. 
Table 1 Comparison of citizens with and without altered liver enzymes

\begin{tabular}{|c|c|c|c|}
\hline & ALE- & ALE+ & $p$-value \\
\hline & $n=1810$ & $n=349$ & \\
\hline Age (years) & $49(41-56)$ & $47(40-55)$ & 0.03 \\
\hline Male sex & $812(44.9 \%)$ & $267(76.5 \%)$ & $<0.001$ \\
\hline Weight (kg) & $72.0(61.0-82.0)$ & $84.0(74.0-95.0)$ & $<0.001$ \\
\hline Height (m) & $1.68(1.60-1.74)$ & $1.73(1.67-1.79)$ & $<0.001$ \\
\hline BMI $\left(\mathrm{kg} / \mathrm{m}^{2}\right)$ & $25.1(22.6-28.1)$ & $27.9(25.4-30.9)$ & $<0.001$ \\
\hline BMI class $(\mathrm{NIH})$ & & & $<0.001$ \\
\hline Underweight & $19(1.0 \%)$ & $0(0.0 \%)$ & \\
\hline Normal & $871(48.1 \%)$ & $66(18.9 \%)$ & \\
\hline Overweight & $607(33.5 \%)$ & $170(48.7 \%)$ & \\
\hline Obesity class 1 & $230(12.7 \%)$ & $81(23.2 \%)$ & \\
\hline Obesity class 2 & $65(3.6 \%)$ & $28(8.0 \%)$ & \\
\hline Obesity class 3 & $18(1.0 \%)$ & $4(1.1 \%)$ & \\
\hline Fatty liver & $637(35.2 \%)$ & $259(74.2 \%)$ & $<0.001$ \\
\hline Fatty liver degree & & & $<0.001$ \\
\hline Light & $428(67.2 \%)$ & 107 (41.3\%) & \\
\hline Moderate & $151(23.7 \%)$ & $102(39.4 \%)$ & \\
\hline Severe & $58(9.1 \%)$ & $50(19.3 \%)$ & \\
\hline Fatty liver type & & & $<0.001$ \\
\hline None & $1173(64.8 \%)$ & $90(25.8 \%)$ & \\
\hline NAFLD & $407(22.5 \%)$ & $160(45.8 \%)$ & \\
\hline AFLD & $230(12.7 \%)$ & 99 (28.4\%) & \\
\hline Waist circumference $(\mathrm{cm})$ & $100.0(93.0-107.0)$ & $105.0(100.0-113.0)$ & $<0.001$ \\
\hline Large waist circumference & $1236(68.3 \%)$ & $259(74.2 \%)$ & 0.028 \\
\hline Glucose (mg/dl) & 89 (83-96) & $93(87-102)$ & $<0.001$ \\
\hline High fasting glucose & $307(17.0 \%)$ & 109 (31.2\%) & $<0.001$ \\
\hline Triglycerides (mg/dl) & $97(68-139)$ & $138(98-206)$ & $<0.001$ \\
\hline High triglycerides & $405(22.4 \%)$ & $159(45.6 \%)$ & $<0.001$ \\
\hline Total cholesterol (mg/dl) & $207(184-234)$ & $215(192-240)$ & 0.005 \\
\hline HDL cholesterol (mg/dl) & $61(51-73)$ & $50(44-61)$ & $<0.001$ \\
\hline Low HDL & $219(12.1 \%)$ & $64(18.3 \%)$ & 0.002 \\
\hline LDL cholesterol (mg/dl) & $126(104-150)$ & $138(117-159)$ & $<0.001$ \\
\hline Systolic blood pressure (mm Hg) & $125(120-140)$ & $130(120-140)$ & $<0.001$ \\
\hline Diastolic blood pressure (mm Hg) & $80(80-90)$ & $85(80-90)$ & $<0.001$ \\
\hline High blood pressure & $1053(58.2 \%)$ & $270(77.4 \%)$ & $<0.001$ \\
\hline Metabolic syndrome & $444(24.5 \%)$ & $171(49.0 \%)$ & $<0.001$ \\
\hline Metabolic syndrome score & & & $<0.001$ \\
\hline 0 & $216(11.9 \%)$ & $19(5.4 \%)$ & \\
\hline 1 & $595(32.9 \%)$ & $59(16.9 \%)$ & \\
\hline 2 & $555(30.7 \%)$ & $100(28.7 \%)$ & \\
\hline 3 & $294(16.2 \%)$ & $97(27.8 \%)$ & \\
\hline 4 & 117 (6.5\%) & 59 (16.9\%) & \\
\hline 5 & $33(1.8 \%)$ & 15 (4.3\%) & \\
\hline
\end{tabular}


Table 1 Comparison of citizens with and without altered liver enzymes (Continued)

\begin{tabular}{llll}
\hline & ALE- & ALE+ & $p$-value \\
& $n=1810$ & $n=349$ & $<0.001$ \\
\hline ALT (U/l) & $20(15-26)$ & $50(44-63)$ & $<0.001$ \\
AST (U/l) & $20(18-24)$ & $33(29-41)$ & $<0.001$ \\
GGT $(\mathrm{U} / \mathrm{l})$ & $17(12-26)$ & $42(27-69)$ & 0.003 \\
Total bilirubin (mg/dl) & $0.60(0.40-0.81)$ & $0.62(0.49-0.90)$ & $<0.001$ \\
Alcohol intake (alcohol units/day) & $2(0-4)$ & $2(1-5)$ & $<0.001$ \\
Wine intake (alcohol units/day) & $2(0-2)$ & $0(0-1)$ & 0.52 \\
Beer intake (alcohol units/day) & $0(0-1)$ & $0(0-1)$ & $<0.001$ \\
Liquor intake (alcohol units/day) & $0(0-0)$ & & \\
\hline
\end{tabular}

Values are given as median (interquartile range) for continuous variables and number (proportion) for dichotomous variables

Abbreviations: ALE altered liver enzymes, BMI body mass index, NAFLD non-alcoholic fatty liver disease, AFLD alcoholic fatty liver disease, NIH National Institutes of

Health, $H D L$ high-density lipoprotein, $L D L$ low-density lipoprotein, ALT alanine transaminase, AST aspartate transaminase, GGT gamma-glutamyl transferase

*Median regression for continuous variables and Pearson's Chi-square test for binary categorical variables

Lastly, alcohol intake was higher in ALE+ than in ALEsubjects.

Table 2 reports the logistic regression models used to investigate the association between FL and potential risk factors.

Model 1 shows that the odds of FL was 5.3 (95\%CI 4.1 to 6.9) times higher in ALE+ than in ALE- citizens. The corresponding probabilities of FL estimated from the logistic regression model are $74 \%$ (95\%CI 70 to $79 \%$ ) for ALE+ and 35\% (95\%CI 33 to 37\%) for ALE- citizens.
Model 2 evaluates whether sex and age are associated with FL independently from ALE. While both sex $(\mathrm{OR}=2.1$, $95 \% \mathrm{CI} 1.7$ to 2.5 for males) and age (OR $=1.8,95 \% \mathrm{CI} 1.6$ to 2.0 per decade) show an independent effect on FL, the OR of ALE changed only slightly (4\%) compared to Model 1.

Model 3, obtained by adding BMI and alcohol intake as predictors to Model 2, shows that BMI is associated with FL $\left(\mathrm{OR}=3.9,95 \% \mathrm{CI} 3.3\right.$ to 4.5 per $\left.5 \mathrm{~kg} / \mathrm{m}^{2}\right)$ with modest changes of the OR of sex $(5 \%)$ and age $(11 \%)$ and with a moderate change of the OR of ALE (23\%).

Table 2 Logistic regression models used to investigate the association between fatty liver and potential risk factors

\begin{tabular}{|c|c|c|c|c|c|c|}
\hline & M1 & M2 & M3 & M4 & M5 & M6 \\
\hline ALE & $5.3^{* *}[4.1$ to 6.9$]$ & $5.1^{* *}[3.9$ to 6.7$]$ & $3.9^{* *}[2.9$ to 5.2$]$ & $4.0^{* *}[3.0$ to 5.4$]$ & $4.2^{* *}[3.2$ to 5.6$]$ & $3.7^{* *}[2.8$ to 5.0$]$ \\
\hline Male & - & $2.1^{* *}[1.7$ to 2.5$]$ & $2.0^{* *}[1.6$ to 2.5$]$ & $2.1^{* *}[1.7$ to 2.6$]$ & $2.0^{* *}[1.6$ to 2.4$]$ & $2.4^{* *}[1.9$ to 3.0$]$ \\
\hline Age (years) / 10 & - & $1.8^{* *}[1.6$ to 2.0$]$ & $1.6^{* *}[1.4$ to 1.8$]$ & $1.5^{* *}[1.4$ to 1.7$]$ & $1.5^{* *}[1.4$ to 1.7$]$ & $1.4^{* *}[1.3$ to 1.6$]$ \\
\hline BMI $\left(\mathrm{kg} / \mathrm{m}^{2}\right) / 5$ & - & - & $3.9^{* *}[3.3$ to 4.5$]$ & - & - & - \\
\hline Alcohol intake (units) & - & - & $1.0[0.9$ to 1.0$]$ & $1.0[1.0$ to 1.1$]$ & - & - \\
\hline Waist circumference $(\mathrm{cm}) / 10$ & - & - & - & $2.5^{* *}[2.3$ to 2.8$]$ & - & - \\
\hline Metabolic syndrome & - & - & - & - & $5.1^{* *}[4.1$ to 6.3$]$ & - \\
\hline Large waist circumference & - & - & - & - & - & $2.9^{* *}[2.3$ to 3.8$]$ \\
\hline High triglycerides & - & - & - & - & - & $3.1^{* *}[2.4$ to 3.9$]$ \\
\hline Low HDL & - & - & - & - & - & $1.6^{*}[1.2$ to 2.2$]$ \\
\hline High blood pressure & - & - & - & - & - & $1.9^{* *}[1.5$ to 2.3$]$ \\
\hline High glucose & - & - & - & - & - & $2.0^{* *}[1.5$ to 2.6$]$ \\
\hline$n$ & 2159 & 2159 & 2159 & 2159 & 2159 & 2159 \\
\hline $\mathrm{AIC}$ & 2750 & 2595 & 2131 & 2244 & 2376 & 2266 \\
\hline $\mathrm{BIC}$ & 2762 & 2618 & 2165 & 2278 & 2405 & 2317 \\
\hline ROC-AUC & 0.61 & 0.72 & 0.83 & 0.81 & 0.79 & 0.81 \\
\hline Pseudo-R² (Nagelkerke) & 0.11 & 0.20 & 0.42 & 0.37 & 0.31 & 0.36 \\
\hline
\end{tabular}

Values are odds ratios and $95 \%$ confidence intervals (logistic regression)

Abbreviations: $M \#$ model number, $A L E$ altered liver enzymes, $B M I$ body mass index, $H D L$ high-density lipoprotein, $A I C$ Akaike information criterion, $B I C$ Bayesian information criterion, $R O C-A \cup C$ area under the ROC curve, pseudo- $R^{2}$ pseudo-squared $\mathrm{R}$

${ }^{*} p<0.01 ;{ }^{* *} p<0.001$ 
Importantly, this model shows that ethanol intake is not associated with FL $(\mathrm{OR}=1.0,95 \% \mathrm{CI} 1.0$ to 1.1 per alcohol unit). All the employed metrics of model fit identified Model 3 as the best of all models (lowest AIC, lowest BIC, highest ROC-AUC and highest pseudo- $\mathrm{R}^{2}$ ).

Model 4, obtained by replacing BMI in Model 3 with $\mathrm{WC}$, shows that $\mathrm{WC}$ is associated with $\mathrm{FL}(\mathrm{OR}=2.5$, 95\% CI 2.3 to 2.8) independently of ALE, sex and alcohol intake. The effect sizes of ALE, sex and age are similar to those of Model 3 using BMI as predictor and ethanol intake is again not associated with FL. (We did not evaluate $\mathrm{BMI}$ and WC together in Model 4 because of the evidence of collinearity as determined by a Belsley-Kuh-Welsch condition number of 31).

Model 5 evaluates the association of MS with FL taking into account ALE, sex and age. Having MS is associated with an odds of FL equal to 5.1 (95\%CI 4.1 to 6.3). (Neither BMI nor WC were entered into this model because WC is already included in the definition of MS and $\mathrm{BMI}$ and $\mathrm{WC}$ were collinear as explained above.)

Model 6 evaluates the independent contribution of each component of the MS (large WC, high triglycerides, low
HDL, high blood pressure and high glucose) to FL. Not only was each component of the MS associated with FL, but all the measures of model fit were better for Model 6 than for Model 5 (lower AIC, lower BIC, higher ROC-AUC and higher pseudo- $\mathrm{R}^{2}$ ), showing that there is some advantage in considering the single components of the MS instead of the MS as its association with FL is concerned.

Figure 2 plots the prevalence of FL in men and women with and without ALE as estimated by Model 3 with ethanol intake set at the median value [32]. In both sexes, the prevalence of FL increases with age and BMI.

\section{Prevalence of and risk factors for NAFLD and AFLD}

The prevalence of NAFLD and AFLD in ALE+ and ALEsubjects was 0.46 ( 0.41 to 0.51$)$ vs. $0.22(95 \% \mathrm{CI} 0.21$ to 0.24 ) and 0.28 ( 0.24 to 0.33 ) vs. 0.13 (CI 0.11 to 0.14 ).

Table 3 reports the multinomial logistic regression models used to investigate the association between FL type and potential risk factors.

Not unexpectedly, all potential risk factors were associated with a lower odds of normal liver vs. NAFLD. All

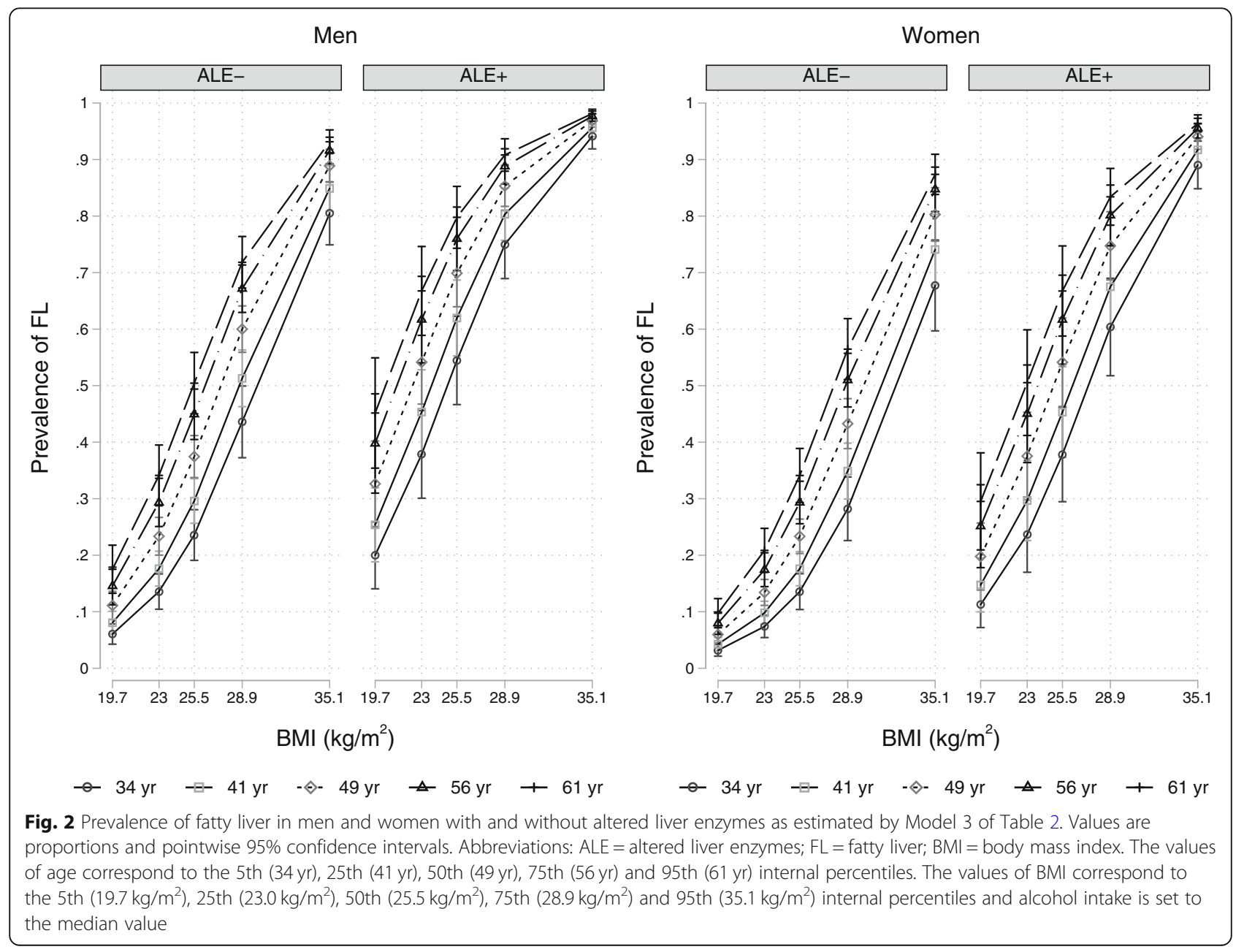


Table 3 Multinomial logistic regression models used to investigate the association between fatty liver type and potential risk factors

\begin{tabular}{|c|c|c|c|c|c|c|}
\hline & M1 & M2 & M3 & M4 & M5 & M6 \\
\hline \multicolumn{7}{|l|}{ Normal liver vs. NAFLD } \\
\hline Altered liver enzymes & $0.20^{* * *}[0.15,0.26]$ & $0.19^{* * *}[0.14,0.25]$ & $0.25^{* * *}[0.18,0.34]$ & $0.24^{* * *}[0.17,0.33]$ & $0.23^{* * *}[0.17,0.31]$ & $0.26^{* * *}[0.19,0.35]$ \\
\hline Male sex & - & $0.61^{* * *}[0.49,0.75]$ & $0.65^{* * *}[0.51,0.82]$ & $0.58^{* * *}[0.46,0.73]$ & $0.64^{* * *}[0.51,0.81]$ & $0.49^{* * *}[0.37,0.64]$ \\
\hline Age (years) / 10 & - & $0.53^{* * *}[0.46,0.60]$ & $0.60^{* * *}[0.52,0.69]$ & $0.61^{* * *}[0.53,0.70]$ & $0.61^{* * *}[0.53,0.70]$ & $0.65^{* * *}[0.56,0.75]$ \\
\hline BMI $\left(\mathrm{kg} / \mathrm{m}^{2}\right) / 5$ & - & - & $0.26^{* * *}[0.22,0.30]$ & - & - & - \\
\hline$W C(\mathrm{~cm}) / 10$ & - & - & - & $0.39^{* * *}[0.34,0.43]$ & - & - \\
\hline Metabolic syndrome & - & - & - & - & $0.20^{* * *}[0.16,0.26]$ & - \\
\hline High waist circumference & - & - & - & - & - & $0.29^{* * *}[0.21,0.39]$ \\
\hline High triglycerides & - & - & - & - & - & $0.38^{* * *}[0.29,0.50]$ \\
\hline Low HDL & - & - & - & - & - & $0.54^{* * *}[0.39,0.76]$ \\
\hline High blood pressure & - & - & - & - & - & $0.56^{* * *}[0.43,0.72]$ \\
\hline High fasting glucose & - & - & - & - & - & $0.52^{* * *}[0.39,0.69]$ \\
\hline$n$ & 1830 & 1830 & 1830 & 1830 & 1830 & 1830 \\
\hline ROC-AUC & 0.61 & 0.72 & 0.83 & 0.82 & 0.78 & 0.81 \\
\hline \multicolumn{7}{|l|}{ AFLD vs. NAFLD } \\
\hline Altered liver enzymes & $1.09[0.81,1.48]$ & $0.89[0.65,1.21]$ & $0.87[0.64,1.20]$ & $0.91[0.66,1.24]$ & $0.87[0.63,1.19]$ & $0.87[0.63,1.20]$ \\
\hline Male sex & - & $1.95^{* * *}[1.44,2.63]$ & $1.96^{* * *}[1.44,2.65]$ & $1.89^{* * *}[1.39,2.56]$ & $1.95^{* * *}[1.44,2.64]$ & $1.52^{*}[1.09,2.13]$ \\
\hline Age (years) / 10 & - & $0.82^{*}[0.70,0.97]$ & $0.83^{*}[0.70,0.97]$ & $0.84^{*}[0.71,0.99]$ & $0.82^{*}[0.69,0.97]$ & $0.80^{*}[0.67,0.96]$ \\
\hline BMI $\left(\mathrm{kg} / \mathrm{m}^{2}\right) / 5$ & - & - & $1.01[0.87,1.17]$ & - & - & - \\
\hline$W C(\mathrm{~cm}) / 10$ & - & - & - & $0.92[0.81,1.03]$ & - & - \\
\hline Metabolic syndrome & - & - & - & - & $1.05[0.79,1.39]$ & - \\
\hline High waist circumference & - & - & - & - & - & $0.68^{*}[0.47,0.97]$ \\
\hline High triglycerides & - & - & - & - & - & $1.58^{* *}[1.17,2.13]$ \\
\hline Low HDL & - & - & - & - & - & $0.64^{*}[0.44,0.93]$ \\
\hline High blood pressure & - & - & - & - & - & $1.10[0.78,1.56]$ \\
\hline High fasting glucose & - & - & - & - & - & $1.12[0.82,1.53]$ \\
\hline$n$ & 896 & 896 & 896 & 896 & 896 & 896 \\
\hline ROC-AUC & 0.51 & 0.60 & 0.60 & 0.61 & 0.61 & 0.63 \\
\hline \multicolumn{7}{|l|}{ Whole model } \\
\hline$N$ & 2159 & 2159 & 2159 & 2159 & 2159 & 2159 \\
\hline $\mathrm{AIC}$ & 3932 & 3754 & 3290 & 3402 & 3537 & 3419 \\
\hline $\mathrm{BIC}$ & 3955 & 3800 & 3347 & 3459 & 3594 & 3521 \\
\hline Pseudo-R ${ }^{2}$ (Nagelkerke) & 0.10 & 0.19 & 0.38 & 0.34 & 0.28 & 0.34 \\
\hline
\end{tabular}

Values are odds ratios and 95\% confidence intervals (multinomial logistic regression). ROC-AUC were calculated for the two binary logistic models underlying the multinomial logistic model

Abbreviations: M\# model number, NAFLD non-alcoholic fatty liver disease, BMI body mass index, WC waist circumference, HDL high-density lipoprotein, AFLD alcoholic fatty liver disease, AIC Akaike information criterion, BIC Bayesian information criterion, ROC-AUC area under the ROC curve, $p s e u d o-R^{2}$ pseudo-squared $\mathrm{R}$ ${ }^{*} p<0.05 ;{ }^{* *} p<0.01 ;{ }^{* * *} p<0.001$

odds ratios were in fact $<1$ for all predictors (Models 16). More interestingly, the same predictors were unable to discriminate AFLD from NAFLD as made clear by the unsatisfactory ROC-curves of the binary ALFD vs. NAFLD model.

In detail, ALE (Models 1-6), BMI (Model 3), WC (Model 4) and MS (Model 5) were not associated with the odds of having AFLD vs. NAFLD. Although male gender and lower age were associated with a greater odds of AFLD vs. NAFLD in all models (Models 1-6), their 95\%CI are wide. It is of some interest that high WC and low HDL made AFLD less likely than NAFLD and that high triglycerides made AFLD more likely than NAFLD (Model 6) but the $95 \% \mathrm{CI}$ of these estimates are again wide.

\section{Discussion}

Although much more epidemiological data are presently available on FL as compared to when the Dionysos Study 
was performed $[3,8]$, there are still few studies performed in representative samples of the general population [10]. FL has a different course in the general population than in primary, secondary and tertiary care centers, where most of the presently available studies on FL were performed [34]. This is in line with the so-called "ecology of medical care" model, according to which only a minority of citizens with a given illness will actually search for and get medical care [14]. Thus, the real burden attributable to FL cannot be estimated without epidemiological data obtained from the general population [15].

The strengths of the present study are that it was performed in a representative sample of the general population, that it enrolled a high number of subjects, and that it built a serum bank that we plan to use in future studies. The most important limitation of the study is the suboptimal response rate (58\%). Although this response rate is the same of the Dionysos Nutrition \& Liver Study [8] and is higher than that reported by most studies [35], it is possible that the citizens who refused to participate to the Bagnacavallo Study differed systematically from those who accepted to participate with an ensuing selection bias. Another limitation of the present study is the use of LUS to diagnose FL. Although LUS is virtually the only feasible option to diagnose FL in population studies, it is known to offer an accurate assessment of FL only starting from an intrahepatic triglyceride content of $10 \%[5,36]$. Thus, lesser degrees of FL may have gone undetected in the present study and our estimates of FL prevalence may be conservative.

In the present study, $74 \%$ of ALE+ citizens had FL compared to $35 \%$ of ALE- citizens. In the Dionysos Nutrition \& Liver Study, performed in the same region of Northern Italy during $2002 / 3,44 \%$ of citizens with suspected liver disease had FL as compared to $35 \%$ of those without suspected liver disease [8]. The estimates made by the Bagnacavallo Study and by the Dionysos Nutrition \& Liver Study are unfortunately not comparable because of the different operational definitions of ALE and suspected liver disease. The criteria for suspected liver disease adopted by the Dionysos Nutrition \& Liver Study did in fact include an altered GGT ( $>35 \mathrm{U} / \mathrm{l}$ ), did not consider AST, and did consider an ALT $>30 \mathrm{U} / \mathrm{l}$ as altered [8]. The Bagnacavallo Study confirms nonetheless, as firstly shown by the Dionysos Nutrition \& Liver Study in a general population [8], that FL is quite common (35\%) among subjects with normal liver enzymes.

The analysis of the potential risk factors for FL yielded two very interesting findings. The first finding is that ethanol intake was not an independent predictor of FL in the general population. The Dionysos Nutrition \& Liver Study reported the same finding even if a direct comparison of the Dionysos Nutrition \& Liver Study and the Bagnacavallo Study is not possible because of the different instruments used to measure alcohol intake [9]. The second finding is that all the components of the MS were associated to FL independently of ALE, gender, age and alcohol intake. The dichotomization implicit in the concept of MS has been criticized by research methodologists on the basis of both clinical and statistical grounds [5, 37]. The findings of the present study offer a further empirical argument for preferring the use of the single components of the MS instead of the whole MS for the study of the association of FL as a whole with cardiometabolic risk factors.

\section{Prevalence of and risks factors for non-alcoholic fatty liver disease and alcoholic fatty liver disease}

Although the present study focused on FL as a whole considering ethanol intake as a potential predictor, we performed an analysis of the prevalence of and the risk factors for NAFLD to allow a comparison with the available studies [10].

In the present study, the prevalence of NAFLD was 46\% among ALE+ and 22\% among ALE- citizens. The corresponding figures for citizens with and without suspected liver disease in the Dionysos Nutrition \& Liver Study were 25 and 20\% [8]. The Bagnacavallo Study and Dionysos Nutrition \& Liver Study estimates are unfortunately not comparable not only because of the different operational definitions of ALE and suspected liver disease as discussed above, but also because the Dionysos Nutrition \& Liver Study employed a different cut-point of alcohol intake to diagnose NAFLD in men $(\leq 20 \mathrm{~g} /$ day) and used a different instrument (7-day prospective diary) to measure ethanol intake [8]. We have, indeed, previously shown that small errors in the estimation of ethanol intake may lead to a substantial difference in the estimated prevalence of NAFLD vs. AFLD [5, 8].

The analysis of the potential risk factors for NAFLD yielded a very interesting finding. All potential risk factors were associated with a lower odds of normal liver vs. NAFLD in all models, which were able to satisfactorily discriminate NAFLD from normal liver. However, the same models were not able to discriminate AFLD from NAFLD. There are several, not mutually exclusive, explanations for this finding. First, the dichotomization of ethanol intake, central to the separation of NAFLD from AFLD [38], could have introduced substantial bias into the inference [37]. Second, we have not cross-validated the recall method used to assess ethanol intake in the present study against an accepted reference method, e.g. the 7-day prospective diary used the Dionysos Nutrition \& Liver Study [8]. Thus, we ignore the measurement error of the method used to assess ethanol intake [39]. This is unfortunately the rule in the literature on $\mathrm{FL}$ and is especially troublesome because the separation of NAFLD from AFLD is based entirely on the dichotomization of 
alcohol intake [38]. Third, it is possible that the separation between NAFLD and AFLD is not relevant at the population level because most of the risk factors for FL as whole are not associated with its separation into NAFLD and AFLD. However, without accurate measurements of ethanol intake, this hypothesis remains highly speculative. In view of the fact that ethanol is a well-known hepatotoxic agent, our data should not be taken as evidence that ethanol intake is not an individual risk factor for fatty liver but simply that with conventional instruments used to detect fatty liver and measure ethanol intake, this relationship was not evident at the population level in Bagnacavallo in 2005/2009.

\section{Conclusions}

In conclusion, FL was highly prevalent in a Northern Italy town in 2005/9 and was more common among ALE individuals. It had no association with alcohol intake but was strongly associated with anthropometry and all the MS components. NAFLD was more common than AFLD but, while anthropometry and all the MS components were able to discriminate normal liver from NAFLD, they did not discriminate AFLD from NAFLD. The cross-sectional data presented in this paper will inform the ongoing and future analyses of the Bagnacavallo cohort, which we hope will offer new and relevant information on the burden of $\mathrm{FL}$ in the general population.

\section{Additional files}

Additional file 1: Table S1. Comparison of the citizens with and without liver ultrasonography among those with altered liver enzymes. In this table we compared the 349 citizens with altered liver enzymes (ALE + ) and with liver ultrasonography (LUS) to the 28 ALE+ citizens without LUS. (DOCX $18 \mathrm{~kb}$ )

Additional file 2: Table S2. Comparison of the citizens with and without liver ultrasonography among those with normal liver enzymes. In this table we compared the 1810 citizens without altered liver enzymes (ALE-) and with liver ultrasonography (LUS) to the 1692 ALE- citizens without LUS. (DOCX $18 \mathrm{~kb}$ )

\section{Abbreviations}

AFLD: Alcoholic fatty liver disease; AIC: Akaike information criterion; ALE: Altered liver enzymes; ALT: Alanine transaminase; anti-HCV: Antibodies against hepatitis C virus; AST: Aspartate transaminase; BIC: Bayesian information criterion; BMI: Body mass index; FL: Fatty liver; GGT: Gammaglutamyl-transferase; HBsAg: Hepatitis B surface antigen; HDL: High-density lipoprotein; LDL: Low-density lipoprotein; LUS: Liver ultrasonography; MS: Metabolic syndrome; NAFLD: Non-alcoholic fatty liver disease; ROCAUC: Area the under the receiver-operating characteristic curve; WC: Waist circumference
\end{abstract}

\section{Acknowledgements}

Not applicable.

\section{Funding}

The authors state that this work has not received any funding.

\section{Availability of data and materials}

The datasets used and/or analyzed during the current study are available from the corresponding author on reasonable request.

\section{Authors' contributions}

FGF, PG and GFS made substantial contributions to conception and design and were the study coordinators. MD, ACDA, FD, AL, FC, SS and GS were involved in generation and collection of data. GB performed statistical analysis. $G B, M B, P A, A G, A C G, C T$, SB were involved in drafting the manuscript or revising it critically. All authors gave final approval of the version to be published.

\section{Ethics approval and consent to participate}

The study was approved by the Ethical Committee of Area Vasta Romagna IRST (reference number 112). All citizens gave written informed consent.

\section{Consent for publication}

Not applicable.

\section{Competing interests}

Stefano Bellantani is an Editorial Board Member for BMC Gastroenterology.

\section{Publisher's Note}

Springer Nature remains neutral with regard to jurisdictional claims in published maps and institutional affiliations.

\section{Author details}

${ }^{1}$ Department of Internal Medicine, Ospedale di Faenza, AUSL Romagna, Faenza, Italy. ${ }^{2}$ Liver Research Center, Italian Liver Foundation, Basovizza, Trieste, Italy. ${ }^{3}$ Department of Medical and Surgical Sciences, University of Bologna, Via Massarenti 9, 40138 Bologna, Italy. ${ }^{4}$ Department of Internal Medicine, Ospedale di Lugo, AUSL Romagna, Locarno, Italy. ${ }^{5}$ Research Center for the Study of Hepatitis, Department of Medical and Surgical Sciences, University of Bologna, Bologna, Italy. ${ }^{6}$ Institute of Clinical Physiology, National Research Council, Pisa, Italy. ${ }^{7}$ Department of Medical Oncology, Istituto Scientifico Romagnolo per lo studio e la cura dei tumori (IRST) IRCCS, Meldola, Italy. ${ }^{8}$ Gastroenterology and Hepatology Service, Clinica Santa Chiara, Locarno, Switzerland.

Received: 12 April 2018 Accepted: 13 November 2018

Published online: 28 November 2018

\section{References}

1. Chalasani N, Younossi Z, Lavine JE, et al. The diagnosis and management of nonalcoholic fatty liver disease: Practice guidance from the American Association for the Study of Liver Diseases. Hepatology. 2018;67:328-57.

2. EASL-EASD-EASO. EASL-EASD-EASO Clinical Practice Guidelines for the management of non-alcoholic fatty liver disease. J Hepatol. 2016;64:1388402.

3. Bellentani S, Saccoccio G, Masutti F, et al. Prevalence of and risk factors for hepatic steatosis in Northern Italy. Ann Intern Med. 2000;132:112-7.

4. Hart CL, Morrison DS, Batty GD, Mitchell RJ, Davey Smith G. Effect of body mass index and alcohol consumption on liver disease: analysis of data from two prospective cohort studies. BMJ. 2010;340:C1240.

5. Bedogni G, Nobili V, Tiribelli C. Epidemiology of fatty liver: an update. World J Gastroenterol. 2014;20:9050-4.

6. Smits MM, loannou GN, Boyko EJ, Utzschneider KM. Non-alcoholic fatty liver disease as an independent manifestation of the metabolic syndrome: results of a US national survey in three ethnic groups. J Gastroenterol Hepatol. 2013;28: 664-70.

7. Lonardo A, Ballestri S, Marchesini G, Angulo P, Loria P. Nonalcoholic fatty liver disease: a precursor of the metabolic syndrome. Dig Liver Dis. 2015;47: 181-90.

8. Bedogni G, Miglioli L, Masutti F, Tiribelli C, Marchesini G, Bellentani S. Prevalence of and risk factors for nonalcoholic fatty liver disease: the Dionysos nutrition and liver study. Hepatology. 2005;42:44-52.

9. Bedogni G, Bellentani S, Miglioli L, et al. The Fatty Liver Index: a simple and accurate predictor of hepatic steatosis in the general population. BMC Gastroenterol. 2006;6:33.

10. Younossi ZM, Koenig AB, Abdelatif D, Fazel Y, Henry L, Wymer M. Global epidemiology of nonalcoholic fatty liver disease-Meta-analytic assessment of prevalence, incidence, and outcomes. Hepatology. 2016;64:73-84. 
11. Suomela E, Oikonen M, Virtanen J, et al. Prevalence and determinants of fatty liver in normal-weight and overweight young adults. The Cardiovascular Risk in Young Finns Study. Ann Med. 2015;47:40-6.

12. van der Voort EA, Koehler EM, Dowlatshahi EA, et al. Psoriasis is independently associated with nonalcoholic fatty liver disease in patients 55 years old or older: Results from a population-based study. J Am Acad Dermatol. 2014;70:517-24.

13. Volzke $H$, Robinson DM, Kleine $V$, et al. Hepatic steatosis is associated with an increased risk of carotid atherosclerosis. World J Gastroenterol. 2005;11: 1848-53.

14. Green LA, Fryer GE, Yawn BP, Lanier D, Dovey SM. The ecology of medical care revisited. N Engl J Med. 2001;344:2021-5.

15. Murray CJ, Lopez AD. Measuring the global burden of disease. N Engl J Med. 2013;369:448-57.

16. Parker R, Hodson J, Rowe IAC. Systematic review: Current evidence in nonalcoholic fatty liver disease lacks relevance to patients with advanced fibrosis. J Gastroenterol Hepatol. 2017;32:950-6.

17. Keogh RH, Cox DR. Case-control studies. Cambridge: Cambridge University Press; 2014.

18. Bellentani S, Tiribelli C, Saccoccio G, et al. Prevalence of chronic liver disease in the general population of northern Italy: the Dionysos Study. Hepatology. 1994;20:1442-9.

19. MacDonald I. Health issues related to alcohol consumption. Oxford: Blackwell Science; 1999.

20. Lohman TG, Roche AF, Martorell R. Anthropometric standardization reference manual. Champaign. Illinois: Human Kinetics Books; 1991.

21. Clinical Guidelines on the Identification, Evaluation, and Treatment of Overweight and Obesity in Adults. The Evidence Report. National Institutes of Health. Obes Res. 1998;6(Suppl 2):51S-209S.

22. Bedogni G, Kahn HS, Bellentani S, Tiribelli C. A simple index of lipid overaccumulation is a good marker of liver steatosis. BMC Gastroenterol. 2010;10:98.

23. Alberti KG, Eckel RH, Grundy SM, et al. Harmonizing the metabolic syndrome: a joint interim statement of the International Diabetes Federation Task Force on Epidemiology and Prevention; National Heart, Lung, and Blood Institute; American Heart Association; World Heart Federation; International Atherosclerosis Society; and International Association for the Study of Obesity. Circulation. 2009:120:1640-5.

24. Sanyal AJ. AGA technical review on nonalcoholic fatty liver disease. Gastroenterology. 2002;123:1705-25.

25. Machado JAF, Parente PMDC, Santos Silva JMC. QREG2: Stata module to perform quantile regression with robust and clustered standard errors. Statistical Software Components, Boston College Department of Economics S457369. 2011; https://ideas.repec.org/c/boc/bocode/s457369.html.

26. Hosmer D, Lemeshow L, Sturdivant R. Applied Logistic Regression. Hoboken: Wiley; 2013.

27. Long JS, Freese J. Regression models for categorical dependent variables using Stata. College Station: Stata Press; 2014.

28. Royston P, Cox NJ. A multivariable scatterplot smoother. Stata J. 2005;5:405-12.

29. Royston P, Sauerbrei W. Multivariable model-building: a pragmatic approach to regression analysis based on fractional polynomials for modelling continuous variables. Chichester: John Wiley; 2008.

30. Harrell F. Regression modeling strategies. Switzerland: Springer International Publishing; 2015.

31. Belsley DA, Kuh E, Welsch RE. Regression diagnostics: identifying influential data and sources of collinearity. New York: Wiley; 1980.

32. Williams R. Using the margins command to estimate and interpret adjusted predictions and marginal effects. Stata J. 2012;12:308-31.

33. Wasserstein R. The ASA's statement on p-values: context, process, and purpose. Am Stat. 2016;70:129-33.

34. Bedogni G, Miglioli L, Masutti F, et al. Incidence and natural course of fatty liver in the general population: the Dionysos study. Hepatology. 2007;46: 1387-91.

35. Morton SM, Bandara DK, Robinson EM, Carr PE. In the 21st Century, what is an acceptable response rate. Aust N Z J Public Health. 2012;36:106-8.

36. Hernaez R, Lazo M, Bonekamp S, et al. Diagnostic accuracy and reliability of ultrasonography for the detection of fatty liver: A meta-analysis. Hepatology. 2011;54:1082-90

37. Greenland S. Invited Commentary: The Need for Cognitive Science in Methodology. Am J Epidemiol. 2017;186:639-45.
38. Kleiner DE, Makhlouf HR. Histology of Nonalcoholic Fatty Liver Disease and Nonalcoholic Steatohepatitis in Adults and Children. Clin Liver Dis. 2016;20: 293-312.

39. Jurek AM, Maldonado G, Greenland S, Church TR. Exposure-measurement error is frequently ignored when interpreting epidemiologic study results. Eur J Epidemiol. 2006;21:871-6.
Ready to submit your research? Choose BMC and benefit from:

- fast, convenient online submission

- thorough peer review by experienced researchers in your field

- rapid publication on acceptance

- support for research data, including large and complex data types

- gold Open Access which fosters wider collaboration and increased citations

- maximum visibility for your research: over $100 \mathrm{M}$ website views per year

At BMC, research is always in progress.

Learn more biomedcentral.com/submissions 